Editorial

\title{
Minimally Invasive Spine Surgery: Does Evidence Favor Minimalism?
}

\author{
Sumit Sinha ${ }^{1}$ \\ ${ }^{1}$ Department of Neurosurgery, All India Institute of Medical Sciences \\ and JPNA Trauma Center, New Delhi, India \\ Indian J Neurosurg 2016;5:67-68.
}

The minimally invasive spine surgery (MISS) is a rapidly developing field with an enormous potential. The main objective of this wonderful technology is to achieve the desired surgical objective with minimal patient morbidity and lesser postoperative complications. It is to be remembered that minimally invasive spine surgery is in itself, not an operative procedure. However, it is related to the access toward achieving an operative goal. Hence, MISS is a tool, albeit not a treatment, for the spinal pathologies and the purported benefit of MISS is probably related to the minimalism it affords in achieving the access and the surgical exposure. The role of MISS has increased tremendously in the last decades with improvements in technology, and initial experiences are being reported for basic procedures such as discectomy and laminectomy. The MISS techniques have been extensively refined recently with modern applications being used for the more advanced spinal fixations and interbody fusions.

The main theoretical benefit of MISS is the excellent panoramic visualization of the offending pathology, however, the two-dimensional vision is the major impediment and henceforth, the steep learning curve for improving the handeye coordination. The minimalistic approach affords preservation of the normal anatomical structures along with a smaller incision, which theoretically should transform into lesser postoperative morbidity, reduced hospital stay, and decreased perioperative blood loss.

MISS has had an enormous impact on the patient outcomes, with patients operated for pathologies such as lumbar disc and lumbar canal stenosis, being discharged on the same day of treatment and these techniques can also be offered to patients with medical comorbidities, morbid obesity, and advanced age. However, in this era of evidence-based medicine, there are increasing concerns of the superiority of MISS over the more conventional standard approaches. There have been many studies in literature regarding the safety and efficacy of MISS techniques. However, comparison studies with good clinical designs have been lacking in the literature. Most of these studies have shown equivalent results in terms of improvement in functional outcome with relatively short, though nonsignificant, perioperative advantages, such as reduced hospital stay with its attendant reduction in hospital costs, lesser perioperative blood loss, and decreased perioperative analgesic usage. The lumbar discectomy was one of the first procedures to be performed using MISS techniques, however, MISS discectomy has a comparative efficacy with standard open approaches in various studies in literature. Arts et $\mathrm{al}^{1}$ conducted a randomized controlled trial comparing tubular discectomy with conventional microdiscectomy. The authors concluded that there was a small but statistically significant improvement in visual analog scale (VAS) scores and functional outcome with standard surgery. However, there was no difference between the perioperative morbidity, recovery rates, and complications among the two approaches. Ryang et $\mathrm{al}^{2}$ compared 60 patients undergoing open microdiscectomy (MD) or MISS procedure. They found small nonsignificant differences favoring MISS in terms of operating time, blood loss, incision length, and complications. No difference was found in VAS scores, Oswestry disability index scores, and the 36-item short form survey (SF-36) scores at a mean follow-up of 16 months. The proponents of MISS procedures relate better functional outcomes to lesser tissue trauma. However, a prospective randomized controlled trial concluded that there was no real clinical advantage of the less traumatized posterior musculature. ${ }^{3}$ Hence, the hypothesis that a less traumatized back muscle leads not
Address for correspondence Sumit Sinha, MS, DNB, MCh, Department of Neurosurgery, All India Institute of Medical Sciences and JPNA Trauma Center, Room No307, Raj Nagar, New Delhi 110029, India (e-mail: sumitaiims@yahoo.com; sumitneuro@gmail.com).
DOI http://dx.doi.org/ 10.1055/s-0036-1586741. ISSN 2277-954X. (c) 2016 Neurological Surgeons' Society License terms of India 
only to a quicker recovery but also to less chronic back pain could not be confirmed. In conclusion, the literature does not appear to favor MISS over the standard MD in cases of unilateral single-level lumbar discectomy. However, there can be small benefit in operating time, blood loss, and incision length.

Similarly, no rigorous prospective controlled trial exists comparing MISS with a standard open laminectomy procedure and there does not appear to be a robust claim in support of MISS as compared with open laminectomy for lumbar canal stenosis. ${ }^{4}$

Hence, a robust clinical evidence favoring MISS over open approaches is lacking and both the procedures result in excellent long-term outcomes with minimal morbidity. There is a need to develop evidence-based recommendations and the studies conducted till now warrant verification in larger prospective registries and well-designed randomized controlled trials in future. The benefit of MISS seems to be more plausible in the more complex procedures associated with exposure-related morbidity. However, at the same time, the operating surgeon should keep in mind that the intended surgical goal should never get jeopardized.

\section{References}

1 Arts MP, Brand R, van den Akker ME, Koes BW, Bartels RH, Peul WC; Leiden-The Hague Spine Intervention Prognostic Study Group (SIPS). Tubular diskectomy vs conventional microdiskectomy for sciatica: a randomized controlled trial. JAMA 2009;302(2):149-158

2 Ryang YM, Oertel MF, Mayfrank L, Gilsbach JM, Rohde V. Standard open microdiscectomy versus minimal access trocar microdiscectomy: results of a prospective randomized study. Neurosurgery 2008;62(1):174-181, discussion 181-182

3 Franke J, Greiner-Perth R, Boehm H, et al. Comparison of a minimally invasive procedure versus standard microscopic discotomy: a prospective randomised controlled clinical trial. Eur Spine J 2009;18(7):992-1000

4 Phan K, Mobbs RJ. Minimally invasive versus open laminectomy for lumbar stenosis: a systematic review and meta-analysis. Spine 2016;41(2):E91-E100 